\title{
MINIMIZAÇÃO DO USO DE ÁGUA E GERAÇÃO DE EFLUENTES ATRAVÉS DO MÉTODO DO DIAGRAMA DE FONTES DE ÁGUA (DFA)
}

Adriano Souza Leão1, Valmir da Cruz de Souza², Edna dos Santos Almeida², Ewerton Emmanuel da Silva Calixto², Fernando Luiz Pellegrini Pessoa ${ }^{2}$

${ }^{1}$ Centro Universitário SENAI CIMATEC, Avenida Orlando Gomes 1845, Salvador-BA; adrianoleaoeng@outlook.com

${ }^{2}$ Centro Universitário SENAI CIMATEC, Avenida Orlando Gomes 1845, Salvador-BA

Resumo: Neste trabalho, analisamos a aplicação do Diagrama de Fontes de Água (DFA), um método de otimização de uso da água em processos industriais, e comparamos com os resultados do balanço de massa realizado por programação matemática do estudo de caso de uma indústria petroquímica brasileira a partir dos mesmos dados. A rede proposta através do DFA no software MINEA 2.0 minimizaria sensivelmente o consumo de água $(<1 \%)$ em relação à integração mássica do artigo de referência e, de forma mais expressiva, reduziria $3 \%$ em relação à planta real em operação; a geração de efluentes, por sua vez, poderia ser minimizada em $63 \%$ e $72 \%$ respectivamente. Embora a diminuição do consumo de água de fontes externas não seria significativa, a rede proposta demandaria alterações estruturais consideravelmente mais simples, geraria menos efluente e, portanto, teria menores custos associados à aquisição, montagem e operação de equipamentos e ao tratamento de correntes residuais bem como menor impacto ambiental.

Palavras-Chave: Reuso; Otimização; Água Industrial

\section{WATER AND WASTEWATER MINIMIZATIONTHROUGH THE WATER SOURCE DIAGRAM (WSD) METHOD}

\begin{abstract}
In this study, we applied the Water Sources Diagram (WSD) method using the MINEA 2.0 software to optimize water use in a petrochemical industry. The data used were obtained from a case study of a plant located in Brazil, which applied a mass balance through mathematical programming. Results showed that the network proposed through the WSD would slightly reduce the water consumption $(<1 \%)$ in comparison to the mass integration of the reference article and, more significantly, minimize 3\% water demand compared to the actual plant in operation. In turn, the generation of wastewater could decrease $63 \%$ and $72 \%$ respectively. Even though the reduction of water demand from external sources would not be significant, the proposed network would require considerably simpler structural changes, generate less effluent and therefore minimize costs of equipment acquisition, assembly and operation of wastewater treatment as well as lessen environmental impacts.
\end{abstract}

Keywords: Reuse; Optimization; Industrial Water 


\section{INTRODUÇÃO}

A indústria é uma atividade antrópica que utiliza água de forma intensiva. 0 consumo de água nas atividades industriais ao redor do mundo equivale a aproximadamente $700 \mathrm{~km}^{3}$ por ano, representando $18 \%$ do total utilizado nas atividades industriais [1]. As propriedades intrínsecas à água a tornam propícia para as mais diversas aplicações na forma de utilidade industrial, além também da assimilação de poluentes direta ou indiretamente pela sua relativa abundância. Entretanto, dada a demanda atual da indústria moderna e seu efeito cumulativo, o risco de escassez tem sido uma preocupação cada vez maior e os custos associados tem aumentado significativamente. O setor produtivo produz significativos volumes de efluentes, que muitas vezes são despejados em corpos hídricos sem nenhum tratamento, degradando o ecossistema e agravando o problema da escassez de água. No que diz respeito ao uso sustentável inerente a água, medidas podem ser tomadas para uma melhor gestão hídrica, tais como mudanças nos processos para redução da demanda e reuso de efluentes aquosos.

No setor petroquímico, consumo de água e geração de efluentes estão associados à ciclos, processos, e equipamentos importantes tais como: caldeiras, torres de resfriamento e refrigeração de equipamentos. O estado da arte aponta para algumas medidas que podem favorecer consideravelmente o uso sustentável da água e redução de custos, que incluem: gestão contínua do uso da água; mudanças de tecnologia e processos; integração de correntes (com reuso, regeneração e reciclo). Alguns métodos matemáticos podem ser utilizados para otimização de uso da água industrial. O Diagrama de Fontes de Água (DFA) é uma das ferramentas que de forma sistemática auxilia na otimização de redes de água industriais, sendo capaz de gerar simultaneamente a meta de mínimo consumo e a rede de transferência de massa.

A aplicação de métodos de otimização do uso da água na literatura competente tem demonstrado que há grande potencial para reutilização de água através de sistemas de otimização baseados em cascata. Os resultados do balanço de massa por programação matemática feito por Hansen et al. [2] mostram que seria possível menor consumo de água de fontes externas e geração de efluentes considerando as restrições heurísticas e estruturais de uma planta petroquímica. A integração proposta poderia reduzir o consumo de água clarificada em 2,6\% e o de água filtrada em 14,3\% em comparação com os dados da planta em operação. Mohammadnejad et al. [3] mostram uma potencial redução de $26 \%$ em uma refinaria de petróleo em Teerã. Os resultados de Mughees e Al-Ahmad [4] apresentam uma diminuição de 38\% o consumo de água captada. Souza et al. [5] mostraram uma redução potencial de 17,61\%.

Demandas significativas e custos associados à água industrial têm direcionado à avaliação de possibilidades de reuso de efluentes tratados ou não. $O$ reaproveitamento de efluentes dentro da própria indústria geradora, ou até mesmo em outras indústrias próximas, tem sido uma alternativa quando viável tecnicamente e vantajosa economicamente. Para este fim, deve-se quantificar e qualificar os efluentes gerados, bem como identificar as operações com entrada de água e as respectivas vazões e qualidades requeridas. Com base nesses dados e na aplicação de métodos de otimização, é possível propor reusos e/ou reciclos [6]. 
Hespanhol [7] recomenda concentrar a fase inicial do programa de reuso de água industrial em sistemas de resfriamento visto que não exigem níveis elevados de qualidade e demandam grandes vazões. Segundo o autor, a água para resfriamento corresponde a $17 \%$ da demanda média de água não potável em indústrias. As possibilidades de reuso trouxeram novas perspectivas para a gestão de água, envolvendo ambas extremidades do processo: demanda/abastecimento de água limpa e geração de águas residuais. Fontes de água com qualidade inferior, tais como efluentes, podem substituir água clarificada em aplicações menos restritivas.

De acordo com Wang e Smith [8], a minimização de efluentes pode ser feita através de reuso e reciclo de água, auxiliados por processos regenerativos ou não, reduzindo custos associado a tratamento. El-Halwagi [9] propôs a divisão das correntes de efluentes em grupos de acordo com suas características e o emprego de tratamentos distintos para cada grupo posteriormente, possibilitando que sejam adotados tratamentos mais específicos e eficientes.

No esquema de reuso, o efluente é utilizado em outra operação sem nenhum tratamento prévio, desde que a concentração de contaminantes seja inferior ao de máximo permitido na entrada da operação de destino (Fig. 1). Normalmente, a carga de contaminantes na corrente permanece inalterada. Desta forma, consegue-se reduzir o consumo de água primária.

Figura 1. Esquema simplificado de uma rede com reuso

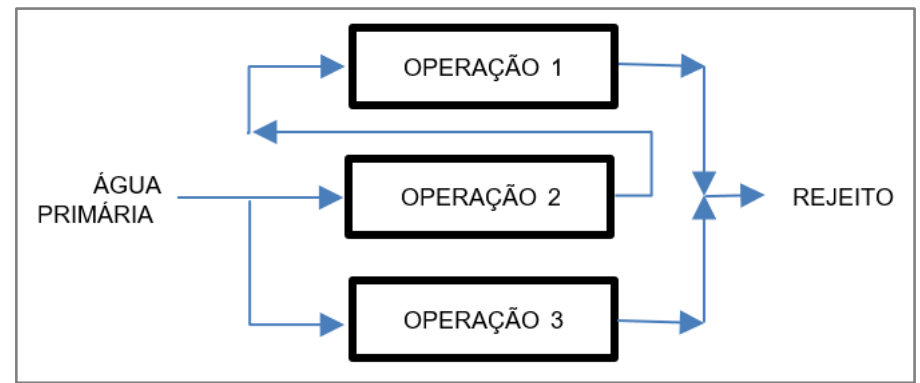

Nesse contexto, o objetivo deste artigo é aplicar o DFA utilizando o software MINEA 2.0 a partir de dados de um estudo de caso realizado por Hansen et al. [2] em uma planta petroquímica brasileira e comparar com os resultados do método de balanço de massa por programação matemática realizado pelos autores.

\section{METODOLOGIA}

O parâmetro Demanda Química por Oxigênio (DQO) foi identificado por Hansen et al. [2] como contaminante mais representativo para otimização do consumo de água nessa indústria petroquímica e confirmado em sua análise do estado da arte. Este contaminante foi utilizado visto que a principal fonte de DQO na indústria estudada são os hidrocarbonetos de petróleo que contaminam as correntes de água durante os processos de resfriamento.

Os dados operacionais (Tab. 1), as concentrações iniciais das fontes externas (Tab. 2), bem como as restrições de reuso da planta (Tab. 3) foram obtidos a partir do 
artigo de referência publicado de Hansen et al. [2]. Os dados operacionais se referem à concentração de entrada e de saída, massa de contaminante e vazões de evaporação, respingo e purga de cada operação (Tab. 1). Para efeitos de cálculo, $1,0 \mathrm{~m}^{3}$ de água é equivalente a 1,0 tonelada de água.

Tabela 1. Dados operacionais

\begin{tabular}{|c|c|c|c|c|c|c|c|c|}
\hline Operação & & $\begin{array}{l}\text { Concent. } \\
\text { entrada } \\
\text { (ppm) }\end{array}$ & $\begin{array}{l}\text { Concent. } \\
\text { Saída } \\
\text { (ppm) }\end{array}$ & $\begin{array}{l}\text { Massa de } \\
\text { contamin. } \\
(\mathbf{g} / \mathbf{h})^{(1)}\end{array}$ & $\begin{array}{c}\text { Evap. } \\
(t / h)\end{array}$ & $\begin{array}{c}\text { Respingo } \\
(t / h)\end{array}$ & $\begin{array}{l}\text { Purga ou } \\
\text { efluente } \\
\text { (t/h) }\end{array}$ & $\begin{array}{l}\text { Vazão de } \\
\text { reposição } \\
(t / h)\end{array}$ \\
\hline Torre resf. 1 & TR1 & 3,7 & 30 & 0 & 629 & 30 & 58 & 717 \\
\hline Torre resf. 2 & TR2 & 4,7 & 25 & 34,6 & 225 & 29 & 23 & 277 \\
\hline Torre resf. 3 & TR3 & 5,8 & 25 & 166,4 & 70 & 9 & 13 & 92 \\
\hline Torre resf. 4 & TR4 & 6,1 & 25 & 220,9 & 34 & 3 & 8 & 45 \\
\hline Resf. equip. 1 & RE1 & 14,7 & 20 & 31,8 & 0 & 0 & 6 & 6 \\
\hline Resf. equip. 2 & RE2 & 3,5 & 20 & 262.5 & 0 & 0 & 15 & 15 \\
\hline
\end{tabular}

Fonte: adaptado de Hansen etal. [2]

A concentração inicial das fontes de água foi considerada igual à menor das "máximas concentrações de entrada" das operações que elas alimentam, considerando a "superestrutura" descrita no estudo que define as restrições de uso e reuso (Tab. 2). As restrições de reuso das correntes da planta (Tab. 3) foram obtidas da "superestrutura".

Tabela 2. Dados das fontes externas

\begin{tabular}{lcc}
\hline \multicolumn{1}{c}{ Fontes externas } & & $\begin{array}{c}\text { Concent. inicial } \\
\text { (ppm) }\end{array}$ \\
\hline Água Clarificada & AC & 3,7 \\
Água de Resf. com Sólidos & AS & 3,7 \\
Água Filtrada & AF & 3,5 \\
\hline Fonte: adaptado de Hansen etal. [2]
\end{tabular}

Tabela 3. Restrições de reuso

\begin{tabular}{lllllll}
\hline Operação & TR1 & TR2 & TR3 & TR4 & RE1 & RE2 \\
\hline TR1 & Não & Sim & Sim & Sim & Sim & Sim \\
TR2 & Sim & Não & Sim & Sim & Sim & Sim \\
TR3 & Sim & Sim & Não & Sim & Sim & Sim \\
TR4 & Sim & Sim & Sim & Não & Sim & Sim \\
RE1 & Não & Não & Não & Não & Não & Não \\
RE2 & Não & Sim & Não & Não & Não & Não \\
\hline
\end{tabular}

Fonte: adaptado de Hansen et al. [2]

O método do Diagrama de Fontes de Água (DFA), apresentado por Gomes [10], é uma das técnicas de integração mássica mais utilizadas se destaca das demais pela sua eficiência, simplicidade e facilidade de aplicação. O método se trata de um procedimento algorítmico heurístico que procura determinar a melhor combinação possível entre as correntes de efluentes aquosos com o objetivo de minimizar o consumo de fonte externa de água e a vazão de efluentes finais. $O$ algoritmo se baseia nos intervalos de concentrações de cada corrente aquosa e na quantidade de massa transferida em cada operação e em cada intervalo. 
O DFA pode otimizar e obter redes mássicas ótimas com um único contaminante ou com múltiplos contaminantes, envolvendo reuso, regeneração com reuso, ou ainda regeneração com reciclo. Pode ser aplicado também à problemas com restrição de vazão, com múltiplas fontes de água, com proibições de reuso e com perdas ou ganhos inerentes ao processo, sem haver a necessidade de modificar a metodologia de cálculo. É possível ainda especificar metas de consumo e gerar a Rede de Transferência de Massa simultaneamente.

O software MINEA foi desenvolvido para automatizar o algoritmo do DFA programado em linguagem $\mathrm{C}$. Pode ser utilizado em problemas envolvendo múltiplos contaminantes e múltiplas operações, permitindo lidar com fontes internas e externas de água e promover reuso, reuso com regeneração e regeneração com reciclo. $O$ MINEA 2.0 é a última versão do programa desenvolvido e fornecido pela Escola de Química da Universidade Federal do Rio de Janeiro. A rede proposta neste trabalho segue o esquema de máximo reuso, onde a as concentrações de entrada e de saída das correntes aquosas em cada operação definem um intervalo de concentração.

Massa de contaminantes transferida para o efluente aquoso:

$\Delta m_{i}=f_{i} *\left(c_{s, i}-c_{e, i}\right)$

Onde $\Delta m_{i}$ representa a variação da massa de contaminante da operação i, $f_{i}$ a vazão da corrente e $c_{s, i}$ e $c_{e, i}$ as concentrações de saída e entrada. Vazão de reposição (make-up):

$f_{\text {reposição }, i}=f_{\text {evaporação }, i}+f_{\text {respingo }, i}+f_{\text {purga }, i}$

Vazão de efluente:

$f_{\text {efluente }, i}=\left[\Delta m_{i} /\left(c_{s, i}-c_{e, i}\right)\right]-f_{\text {reuso }, i}$

\section{RESULTADOS E DISCUSSÃO}

Os resultados obtidos pela otimização do DFA através do software MINEA 2.0 mostram que o consumo de fontes externas de água seria da mesma ordem de grandeza dos obtidos pela integração mássica feita por Hansen et al. [2] (Tab. 4). As fontes de água diferiram tanto em volume consumido quanto na origem. Verificou-se que uma vazão menor seria reusada pelas operações internamente. No entanto, 0 consumo de água total por fontes externas seria minimizado em 2,5 t/h.

Tabela 4. Resultados do DFA no MINEA 2.0

\begin{tabular}{lccc|cccccc}
\hline \multirow{2}{*}{ Operação } & \multicolumn{3}{c|}{ Fontes externas (t/h) } & \multicolumn{6}{c}{ Fontes Internas (t/h) } \\
\cline { 2 - 10 } & AC & AS & AF & TR1 & TR2 & TR3 & TR4 & RE1 & RE2 \\
\hline TR1 & 703 & 14 & - & - & - & - & - & - & - \\
TR2 & 260,5 & - & - & - & - & - & - & - & - \\
TR3 & 83 & - & - & - & - & - & - & - & - \\
TR4 & 40 & - & - & - & - & - & - & - & - \\
RE1 & 2 & - & - & - & - & - & - & - & - \\
RE2 & - & - & 15 & - & 15 & - & - & - & - \\
\hline \multirow{2}{*}{ Total } & $\mathbf{1 0 8 8 , 5}$ & $\mathbf{1 4}$ & $\mathbf{1 5}$ & $\mathbf{0}$ & $\mathbf{1 5}$ & $\mathbf{0}$ & $\mathbf{0}$ & $\mathbf{0}$ & $\mathbf{0}$ \\
\hline
\end{tabular}


Para calcular a vazão de efluentes, utilizamos os resultados das concentrações e de reuso geradas pelo DFA (Tab. 5) e as massas de contaminantes da Tabela 1 através da Equação 3. A Figura 2 apresenta os resultados do diagrama de rede proposto através do DFA no MINEA. Verificou-se que, em termos de estrutura, 0 diagrama de rede proposto teria menos misturadores do que a rede proposta por Hansen et al. [2], reduzindo de 4 reduziu para 2. Como consequência, haveriam menores custos associados à reestruturação da rede, como na aquisição, montagem e operação de novas tubulações e misturadores, além de menor vazão para tratamento ou readequação.

Tabela 5. Cálculo das vazões de efluentes partir dos resultados do DFA

\begin{tabular}{lcccc}
\hline Operação & $\begin{array}{c}\text { Concent. entrada } \\
\text { DFA (ppm) }\end{array}$ & $\begin{array}{c}\text { Concent. saída } \\
\text { DFA (ppm) }\end{array}$ & $\begin{array}{c}\text { Reuso DFA } \\
(\mathbf{t} / \mathbf{h})\end{array}$ & $\begin{array}{c}\text { Efluente } \\
\text { (t/h) }\end{array}$ \\
\hline TR1 & 3,7 & 30 & - & 0,0 \\
TR2 & 4,59 & 24,9 & - & 1,7 \\
TR3 & 3,7 & 22,9 & - & 8,7 \\
TR4 & 3,7 & 22,6 & - & 11,7 \\
RE1 & 3,7 & 9 & - & 6,0 \\
RE2 & 3,5 & 20 & 15 & 0,9 \\
\hline
\end{tabular}

Os resultados globais compilados na Tabela 6 mostram que seria necessário ligeiramente menos água de fonte externa $(-0,22 \%)$ em relação à integração mássica de Hansen et al. [2], porém, esse resultado não é significativamente diferente. Quanto aos dados da planta real em operação, poderia haver uma redução de $3 \%$ de consumo de água de fontes externas.

Tabela 6. Comparação entre resultados

\begin{tabular}{lccc}
\hline Operação & $\begin{array}{c}\text { Dados } \\
\text { operacionais }{ }^{(1)}(\mathbf{t} / \mathbf{h})\end{array}$ & $\begin{array}{c}\text { Integração } \\
\text { mássica }{ }^{(1)}(\mathbf{t} / \mathbf{h})\end{array}$ & $\begin{array}{c}\text { Resultados do } \\
\text { DFA (t/h) }\end{array}$ \\
\hline Fontes externas & & & 1088,5 \\
\hline Consumo de AC & 1131 & 1088 & 14 \\
Consumo de AS & 21 & 14 & 15 \\
Consumo de AF & 1152 & 18 & 1117,5 \\
\hline Total & 0 & 1120 & 15 \\
\hline Fontes internas & 105 & 21 & 29 \\
\hline Geração de efluentes & & 78 & \\
\hline
\end{tabular}

(1) Fonte: adaptado de Hans en etal. [2]

Considerando a planta operando $24 \mathrm{~h} / \mathrm{dia}$, isso poderia resultar em uma economia de aproximadamente 24,8 toneladas de água externa consumida por mês e 298.000 toneladas por ano. A geração de efluentes poderia ser minimizada em cerca de $63 \%$ em relação aos resultados do artigo de referência e $72 \%$ em relação aos dados da planta em operação. 
Figura 2. Diagrama de rede do DFA

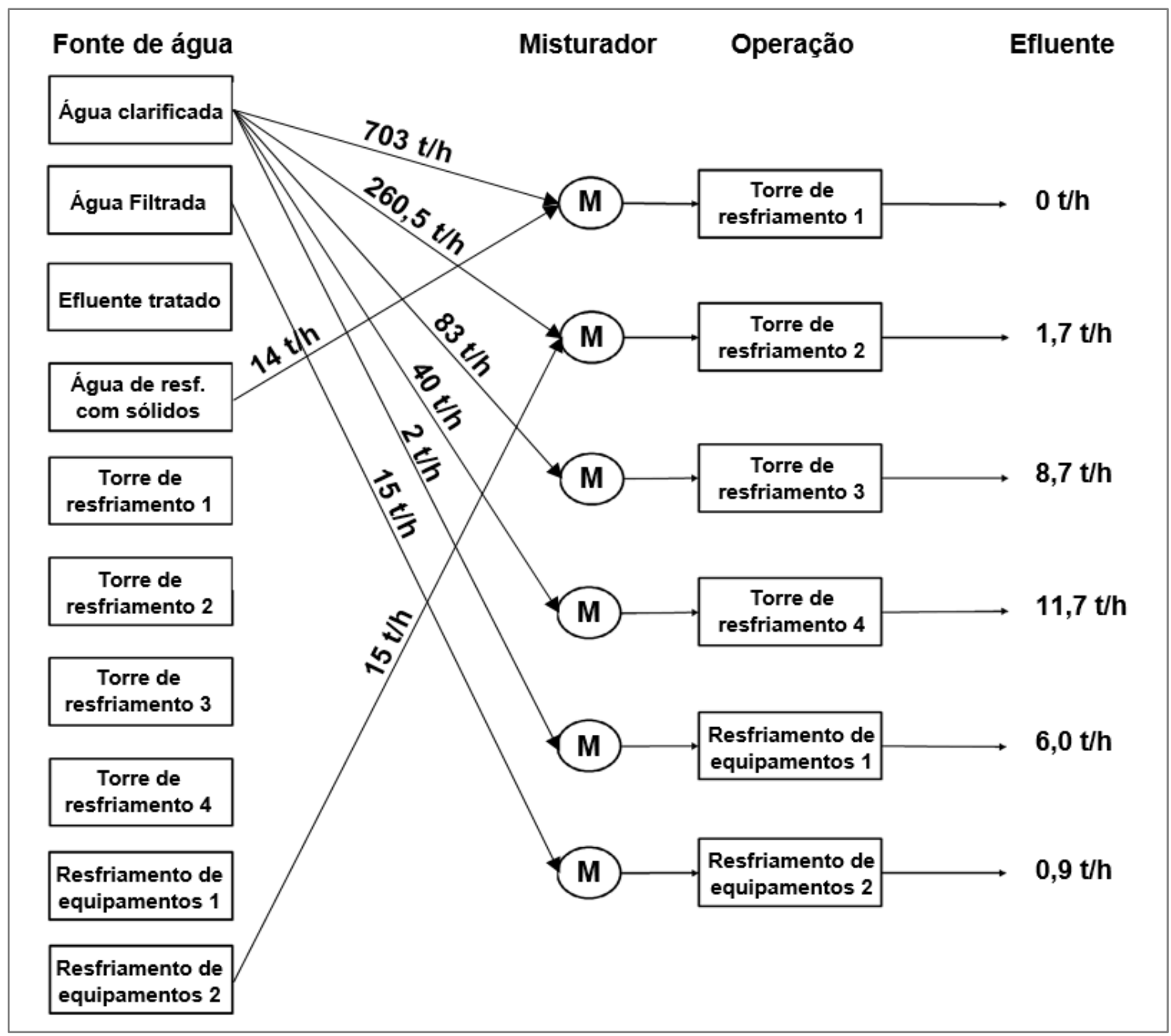

\section{CONCLUSÃO}

Este estudo demonstra o potencial de otimização do uso da água através do método DFA com o software MINEA 2.0 tendo em vista uma redução no consumo de fontes externas e na geração de efluentes em uma indústria petroquímica. A rede proposta neste artigo possui algumas vantagens, principalmente estruturais, em relação aos resultados do artigo de referência [2], legitimando a funcionalidade e aplicabilidade do método. Comparativamente, observou-se que a minimização no consumo de água de fontes externas não seria numericamente significativa. Entretanto, outros benefícios podem ser destacados, começando pela menor geração de efluentes, que aliada a uma pequena redução no consumo global de água limpa, corroboraria com um menor impacto ambiental gerado pela planta no que se refere à escassez e poluição da água. Adicionalmente, menores custos e infraestrutura estariam associados ao tratamento ou readequação dessas correntes residuais. Quanto às modificações estruturais para implementação da rede proposta, seriam necessárias alterações consideravelmente mais simples e, consequentemente, menores custos de investimento associados à aquisição de tubulações e misturadores, bem como na montagem e operação desses equipamentos. 


\section{Agradecimentos}

Agradecimento especial a EMBRAPII, que patrocinou a bolsa de mestrado referente ao projeto Curtain Wall - Tecnored para o pesquisador/autor deste artigo.

\section{REFERÊNCIAS}

1 QUAGLIA, A.; et al. Industrial process water treatment and reuse: a framework for synthesis and design. Ind. Eng. Chem. Res. 53, 5160e5171. 2014.

2 HANSEN, E. et al. Water and wastewater minimization in a petrochemical industry through mathematical programming. Journal of Cleaner Production, v. 172, p. 18141822. 2018.

3 MOHAMMADNEJAD, S. et al. Water pinch analysis for water and wastewater minimization in Tehran oil refinery considering three contaminants. Environ. Monit. Assess. 184, 2709 e 2728. 2012.

${ }^{4}$ MUGHEES, W.; AL-AHMAD, M. Application of water pinch technology in minimization of water consumption at a refinery. Computers \& Chemical Engineering, v. 73, p. 34-42, 2015.

5 Souza, A.A. Application of Water Source Diagram (WSD) method for the reduction of water consumption in petroleum refineries. Resour. Conserv. Recycl. 53. 2009.

6 FARIA, D.C. Reuso das Correntes de Efluentes Aquosos em Refinarias de Petróleo. 246 f. Dissertação (Mestrado em Engenharia Química) - Centro Tecnológico, Universidade Federal de Santa Catarina, Florianópolis. 2004.

7 HESPANHOL, I. Potencial de Reuso de Água no Brasil: Agricultura, Indústria, Municípios, Recarga de Aquíferos. Bahia Análise \& Dados, v. 13, n. especial, p. 411437. 2003.

8 WANG, Y.P.; SMITH, R. Wastewater Minimisation. Chemical Engineering Science, v. 49, n. 7, p. 981-1006. 1994.

9 EL-HALWAGI, M.M. Pollution Prevention Through Process Integration: Systematic Design Tools. San Diego: Academic Press. 1997.

10 GOMES, J.F.S. Procedimento para Minimização de Efluentes Aquosos. $228 \mathrm{f}$. Dissertação (Mestrado em Tecnologia de Processos Químicos e Bioquímicos) Escola de Química, Universidade Federal do Rio de Janeiro, Rio de Janeiro. 2002. 